\title{
Automatic Coronary Artery Detection on in Situ Heart Images
}

\author{
EU Schirmbeck', I Nagy², H Mayer ${ }^{2}$, A Knoll $^{2}$, \\ R Lange ${ }^{1}$, R Bauernschmitt ${ }^{1}$ \\ 'German Heart Center Munich, Clinic for Cardiovascular Surgery, Technische Universität München, \\ Munich, Germany \\ ${ }^{2}$ Technische Universität München, Fakultät für Informatik, Robotics and Embedded Systems, \\ Munich, Germany
}

\begin{abstract}
Minimally invasive and endoscopic operations became routine in heart surgery. Unlike the conventional operating procedures these techniques cause minor lesions to the patients. The minimized incisions lead to fewer postoperative complications and a rapid recovery. However it remains difficult to operate with endoscopic instruments through a keyhole and perform a complex procedure. In cardiac surgery coronary artery bypass grafting is a standard procedure which is also performed endoscopically. The identification of the target artery is the most important and a sophisticated process directly in the beginning of the surgery. We present an intraoperative tool for the surgeon to clearly identify the arteries visible in the endoscope view.
\end{abstract}

\section{Introduction}

In minimally invasive coronary artery bypass graft operations the exact identification of the target artery on the heart surface persists complex. The benefits of minimally invasive and endoscopic cardiac surgery are manifold, including less trauma to tissue and consequently less pain, shorter hospital stay and faster recovery. Nevertheless problems remain in the complex operative procedure with long instruments and small aditus to the operating field. The combination of the real endoscopic camera view and preoperative diagnostic images in one augmented-reality-model facilitates the identification and classification of the coronary arteries on the heart surface. This approach combines several advanced techniques into a single tool allowing real-time augmentation of endoscopic beating heart image sequences with the corresponding angiogram assisting the surgeon in classifying the arteries rapidly and reliably. The exact identification of the target arteries is difficult not only in open heart surgery [1] but also in endoscopic or minimally invasive heart surgery due to the limited view through the endoscopic camera. The preoperative coronary angiography illustrates the position and the course of the coronary arteries.

The goal is to provide a set of operation room suitable software tools which allow the analysis of angiograms in a user friendly manner. The software functionality consists of an exact match of pre-operatively recorded angiograms with the visible in situ coronary arteries with a semi-transparent cross-fade view of the angiogram.

The visible part of the coronary tree as shown in figure 1 on the left hand side has to be matched with the highlighted part on the right side, allowing to find even covered runs of the arteries (the enclosing lines are highlighted by hand for illustration purposes).

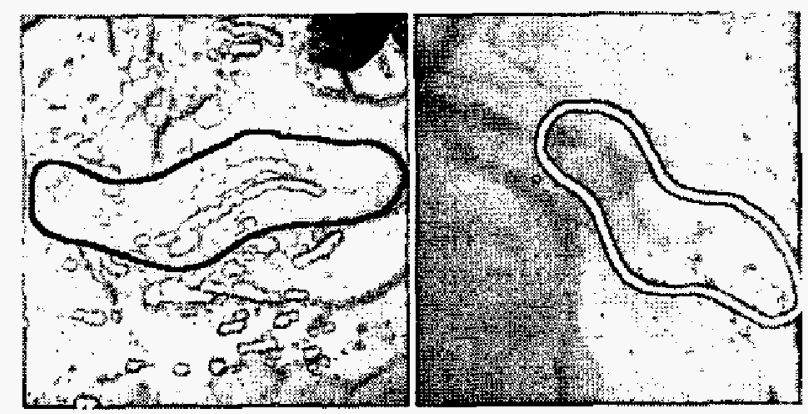

Figure 1. View of the heart surface and corresponding angiogram with marked coronary artery.

\section{Methods}

The preoperatively drawn up angiograms and the in situ heart image sequences are matched with the real intraoperative endoscope images in one augmented reality model. Matching coronary arteries to their corresponding angiogram requires segmenting the images and presenting the results in a symbolic illustration, which facilitates the matching process.

The angiograms and the in situ heart image sequences are digitally available. The heart images are in a $720 \times 576$ 24 bit RGB format, while the angiograms are available as $512 \times 5128$ bit greyscale images. 


\subsection{The in situ heart images}

The segmentation of the coronary arteries is performed to distinguish the vessels from the background in a colourbased technique. The significant parts of the heart surface, which are up for segmentation, are damaged by specular reflection (fig. 2a). Specular reflections occur as a result of light reflections on glossy surfaces. Due to their high intensity a thresholding operator is sufficient to localize the glossiness (fig. 2b). In 24 bit RGB images almost each of the available colour channels (red, green, blue) can be used for thresholding, even a computed 8 bit greyscale channel is suitable. Dilating of the thresholded areas is required caused by artefacts in form of a 1-2 pixel wide dark contour around a specular reflection. The underlying image colours are reconstructed using a fill-in technique based on chain coding $[2,3]$.

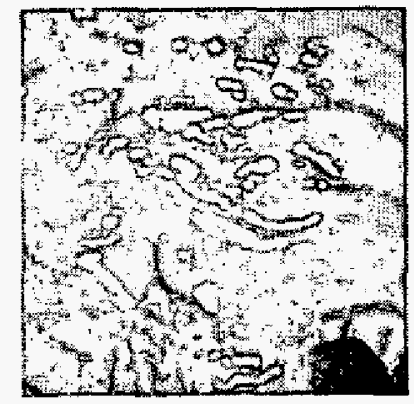

Figure 2a. Heart suface.

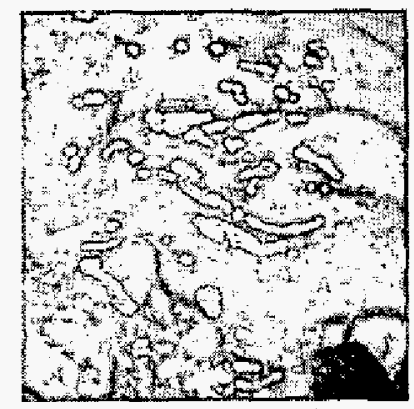

Figure $\mathbf{2 b}$. Thresholding operator.

\subsection{Segmentation of the angiogram}

An efficient implementation of a Gauß/Sobel filter combination detects not only the runs of the vessels (fig. 3a) but also their thickness (fig. 3b). Features of a chain coded representation show additional advantages of the matching process with the (likewise chain coded) coronary arteries for the symbolic representation of the segmented parts $[4,5]$.
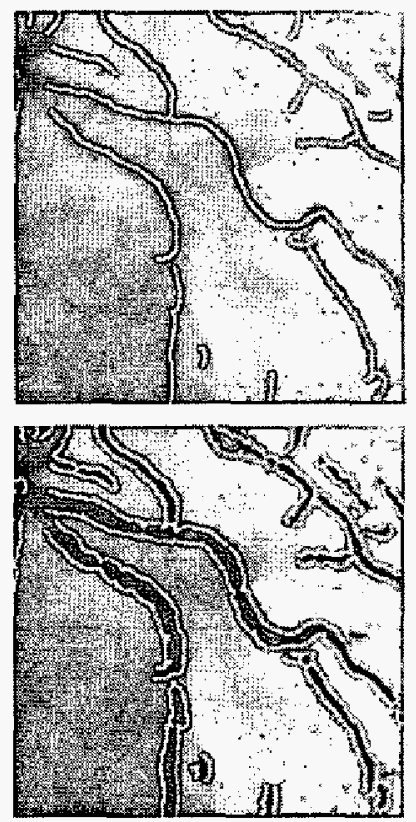

Figure 3. Angiogram segmentation with the course (above) and outline figure of the coronary arteries (below).

\subsection{Augmentation}

In order to achieve a more realistic augmentation a Mass-Spring-NURBS based on soft tissue simulation avails as framework for the angiographic view [6]. The heart image and the corresponding angiogram are mapped to an OpenGL textured "canvas" [7]. The canvas of the heart image is a simple textured polygon; the angiogram represents a textured NURBS surface. Figure 4 illustrates a textured NURBS-surface to a mass-spring grid. The mass points are simultaneously control points of the freeform surface.

The arrangement and the number of the mass/control points are not random, since they are closely related to the parameters (knot sequence, order) of the surface. The interconnection between masses by springs is done using a Delaunay triangulation: the masses are arbitrary points in 3D Cartesian space and the sides of the resulting triangles are springs. The most challenging task is to fix the mass/control points to the underlying heart surface on natural landmarks. 


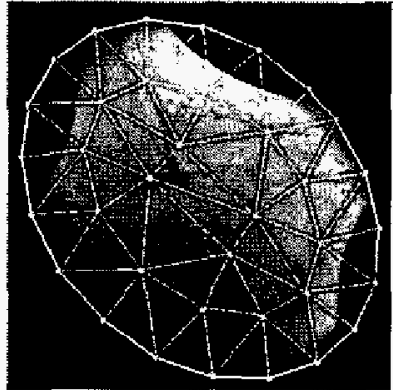

Figure 4. M-S-NURBS model of the surface.

\subsection{Matching}

To find the best fitting angiogram to the currently visible part of a coronary artery, a search of similarity is obligatory. Both modalities (angiogram and heart image sequence) in a symbolic representation enable to find the best match to compute the transformation parameters (axis for flipping, translation and rotation angle) [8].

\section{Results}

A virtual soft tissue simulation is implemented which represents the exact identification of the target coronary artery on the heart surface in the endoscopic cardiac procedure.

Figure 5 shows the result of the specular reflection removal; the approach surpasses real-time ( 30 frames per second) requirements on high resolution colour images (512 x 51224 bit RGB).

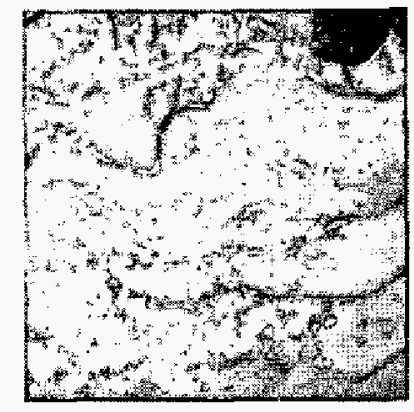

Figure 5. Removed specularities

By flipping the angiography horizontally and by rotating the angiogram best fitting to the section of the real heart surface is found.

Real-time matching of angiographies with in situ heart image sequences permits an exact classification of coronary arteries rapidly and reliably. The endoscopic view is shown in figure 6 .

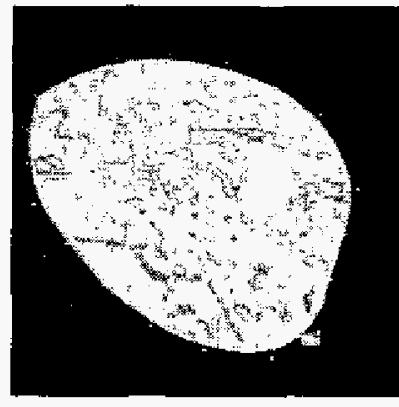

Figure 6. Augmented and crossfaded view.

The live heart image sequence is augmented by a semitransparent crossfaded view of the angiogram.

\section{Discussion and conclusions}

The new tool-set of augmented real-time live camera images extends the intraoperative view to identify exactly the coronary arteries. The surgeon is assisted by an approach to enhance and ameliorate the success of an endoscopic operation like telemanipulated assisted interventions. No additional image acquisition or intraoperative angiography is needed.

The major part of the system is implemented, nevertheless further upgrades on two important issues queue: a refining of the matching process and the advancement of fixing the mass/control points to the underlying heart surface.

\section{Acknowledgements}

This work was supported in part by the German Research Foundation (DFG) within the Collaborative Research Centre SFB 453 on "High-Fidelity Telepresence and Teleaction".

\section{References}

[1] Banerjee S, Crook AM, Dawson JR, Timmis AD, Hemingway $H$. Magnitude and consequences of error in coronary angiography interpretation (the ACRE study). Am J Cardiol 2000;1;85(3):309-14.

[2] Freeman H. Techniques for the digital computer analysis of chain-encoded arbitrary plane curves. Proc Natl Elect Conf 1961;17:421-432.

[3] Kuhl FP, Giardina CR. Elliptic Fourier features of closed contours. Computer graphics and image processing 1982;18:236-258.

[4] Toledo R, Orriols X, Radeva P, Binefa X, Vitria J, Canero C. Villanueva JJ. Eigensnakes for Vessel Segmentation in Angiography. International Conference on Pattern Recognition 2000; Barcelona, Spain.4:4340. 
[5] Yim PJ, Choyke PL, Summers RM. Greyscale skeletonization of small vessels in magnetic resonance angiography. IEEE Transactions on Medical Imaging 2000;6:568-576.

[6] Kühnapfel U, Cakmak HK, Maass H. Endoscopic Surgery Training using Virtual Reality and deformable Tissue Simulation. Computers \& Graphics 2000;24:671-682.

[7] Wernecke J. Programming Object-Oriented 3D Graphics with Open Inventor in Addison Wesley (Ed). The Inventor Mentor; 1999.
[8] Cottin S, Delingette H, Ayache N. Real-Time Elastic Deformations of Soft Tissues for Surgery Simulation. IEEE Transactions on Visualization and Computer Graphics 1999;5(1):62.

Address for correspondence.

Eva U. Schirmbeck.

German Heart Center Munich.

Clinic for Cardiovascular Surgery.

Lazarettstr. 36.

D-80636 Munich, Germany.

schirmbeck@dhm.mhn.de. 